Zum Mechanismus von Kontakteliminierungen

\title{
XIV. Die Produktverteilungen an einigen Sulfatkatalysatoren für verschiedene Butanderivate *
}

\author{
P. Andréu, M. Rosa-Brusin, C. Sánchez und H. Noller ** \\ Escuela de Química, Universidad Central de Venezuela, Caracas
}

(Z. Naturforschg. 22 b, 814-819 [1967] ; eingegangen am 9. Februar 1967)

\begin{abstract}
Mit den in der vorhergehenden Mitteilung untersuchten Reaktionssystemen wurde unter denselben Bedingungen wie dort die Produktverteilung, d.h. das Verhältnis der drei linearen Butene, bestimmt. Mit zunehmender Temperatur nähert sich diese Verteilung zunehmend an das Gleichgewicht an.

Mit abnehmender Temperatur nehmen die Abweichungen von der Gleichgewichtsverteilung zu, und zwar wird häufig cis-2-Buten vor trans-2-Buten bevorzugt. Diese cis-Selektivität scheint mit abnehmender Aktivität des Katalysators größer zu werden.

Die Produktverteilung mit 1-Chlor- und 1-Brombutan ist der mit 2-Chlor- und 2-Brombutan erhaltenen sehr ähnlich. Lediglich $\mathrm{Li}_{2} \mathrm{SO}_{4}$ macht eine Ausnahme. Im unteren Bereich des Temperaturintervalls erhält man ausschließlich 1-Buten.

Ein Vergleich der Produktverteilung aus Eliminierungs- und Isomerisierungs-Versuchen läßt den Schluß zu, daß die Produktverteilung der Eliminierung, zumindest zu einem ganz erheblichen Teil, die Primärverteilung sein muß und nicht auf einer nachträglichen Isomerisierung beruht.
\end{abstract}

Diese Arbeit schließt sich unmittelbar an die vorhergehende an. Katalysatoren, Reaktanten und Experimentiertechnik sind die gleichen.

Für mechanistische Studien eignet sich neben Kinetik, Aktivität, Arrhenius-Parametern und weiteren Eigenschaften insbesondere auch die Produktverteilung. Vor allem die Anwendung der Gaschromatographie-Technik macht es möglich, diese so wichtige Informationen vermittelnde Eigenschaft zu untersuchen. Natürlich ist dabei nicht die dem Gleichgewicht entsprechende Endverteilung interessant, sondern insbesondere die Verteilung, die entsteht, wenn die Molekel einmal und noch nicht öfter reagiert hat, d. h. die Verteilung, die nach der ersten zur Reaktion führenden Adsorption der Molekeln entsteht, und von der aus sich dann in einer Reihe von Folgereaktionen das Gleichgewicht einstellt.

Diese Verteilung kann man Primärprodukt-Verteilung nennen. $\mathrm{Da} \beta$ diese gerade der Gleichgewichtsverteilung entspricht, scheint relativ selten zu sein, und dies leuchtet bei näherer Betrachtung auch ein. Betrachten wir eine Molekel A, die die Produkte B und $\mathrm{C}$ bilden kann. Die Gleichgewichtsverteilung zwischen zwei Produkten B und C wird durch den Unterschied der freien Energien der beiden Pro-

* XIII. Mitteilung; vorhergehende Arbeit, Z. Naturforschg. 22 b, 809 [1967]. dukte bestimmt. Die Primärprodukt-Verteilung wird, zumindest näherungsweise, durch den Unterschied der (freien) Aktivierungsenergien der beiden zu B und C führenden Reaktionswege bestimmt. Es ist höchst unwahrscheinlich, und wäre wirklich ein seltsames Spiel des Zufalls, würden diese beiden Differenzen ganz genau gleich sein. So wird man im allgemeinen immer eine vom Gleichgewicht verschiedene Primärprodukt-Verteilung erwarten. Natürlich fällt dieses Phänomen besonders dann in die Augen, wenn ein thermodynamisch weniger stabiles Produkt primär bevorzugt wird. Dies ist jedoch nur eine der verschiedenen denkbaren Möglichkeiten.

\section{Experimenteller Teil}

Die Experimente wurden wie im vorhergehenden Teil durchgeführt und in der Regel zusammen mit diesen. Die einzige Änderung betrifft die Trennsäule. Es konnte kein Trennmaterial gefunden werden, das ohne Temperaturprogramm die Trennung der drei linearen Butene voneinander sowie von den Reaktanten ermöglicht hätte. Das Verhältnis der drei linearen Butene wurde darum in einem von der Aktivitätsbestimmung getrennten Arbeitsgang ermittelt.

** Anschrift auch: Physikalisch-Chemisches Institut der Universität München. 


\section{Trennsäule}

Trennmaterial: a) $30 \mathrm{Gew} . \%$ Dinonylphthalat auf Chromopak 60-80mesh; Curtin USA; b) 30 Gew.-\% Dimethylsulfolan auf Chromosorb 30-60 mesh, Curtin USA. Die sonstigen Bedingungen für beide Säulen waren gleich. Abmessungen: $3,5 \mathrm{~m} \cdot 1 / 4$ Zoll. Temperatur: $50{ }^{\circ} \mathrm{C}$. Trägergas: $120 \mathrm{ml} \mathrm{He} /$ Minute. Reihenfolge der Piks: 1-Buten, trans-2-Buten, cis-2-Buten. Die Reaktanten werden von den Säulen nicht durchgelassen.

\section{Identifikation der Piks}

Dazu wurden Muster, bezogen von der Firma Air Products and Chemicals USA, injiziert.

Die verwandten Säulen vermögen nicht 1-Buten und Isobuten zu trennen. $\mathrm{Da}$ jedoch, abgesehen von $\mathrm{Li}_{2} \mathrm{SO}_{4}$, die Menge an 1-Buten stets relativ klein war, so wäre, falls es überhaupt entstünde, auch die Menge an Isobuten klein. Bei früheren Untersuchungen, bei denen wir auf Isobuten prüften, konnten wir nie welches auf- finden. Sein Auftreten ist unter den hier vorliegenden Bedingungen zumindest unwahrscheinlich.

Wie wir kürzlich feststellten ${ }^{1}$, entsteht Isobuten beim Cracken von (formal gleich Benzoleliminierung aus) 1- und 2-Phenylbutan am Silicium-Aluminiumoxid-Crackkatalysator, jedoch erst bei Temperaturen über $400{ }^{\circ} \mathrm{C}$. Auch dieser Befund macht die Entstehung von Isobuten unter den hier vorliegenden Bedingungen unwahrscheinlich.

\section{Berechnung der Produktverteilung}

Dazu wurde, unabhängig vom jeweiligen Umsatz, die Summe der Pikflächen der drei Butene gleich $100 \%$ gesetzt und der prozentuale (Flächen-) Anteil der einzelnen Butene errechnet.

\section{Resultate und Diskussion}

Die Produktverteilungen sind aus der Tabelle zu ersehen. Die Abbn. 1 bis 5 stellen einige Beispiele

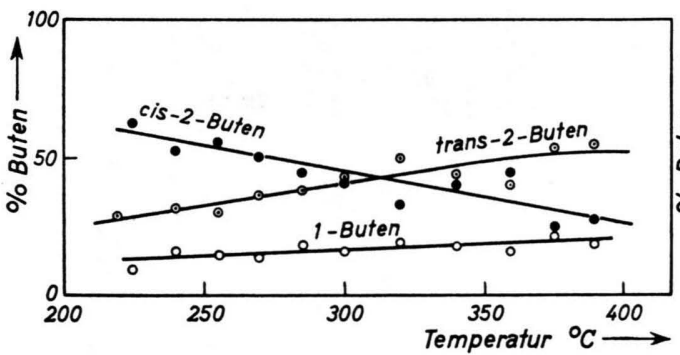

Abb. 1. Produktverteilung für 2-Brombutan an $\mathrm{Li}_{2} \mathrm{SO}_{4}$.

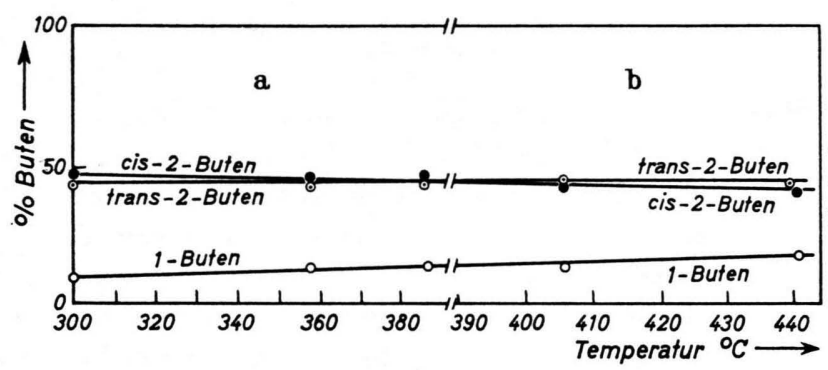

Abb. 3. Produktverteilung an $\mathrm{K}_{2} \mathrm{SO}_{4}$, a) für 2-Brombutan, b) für 1-Brombutan.

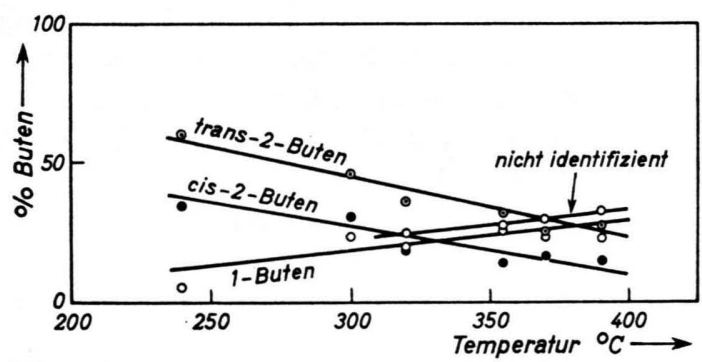

Abb. 5. Produktverteilung für 1-Brombutan an $\mathrm{Al}_{2}\left(\mathrm{SO}_{4}\right)_{3}$.

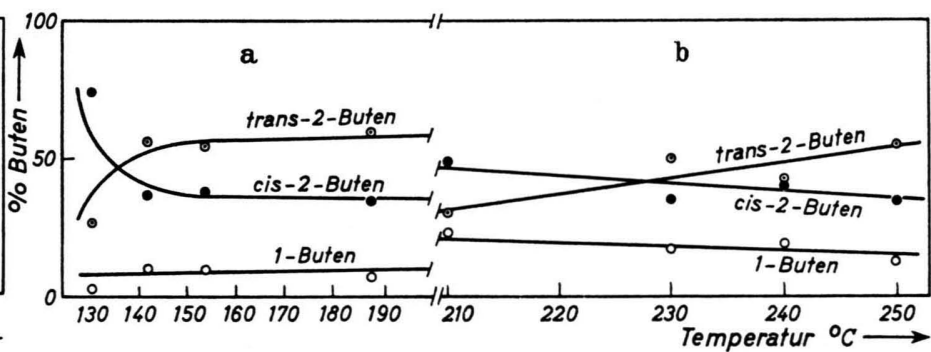

Abb. 2. Produktverteilung an $\mathrm{BaSO}_{4}$, a) für 2-Brombutan, b) für l-Brombutan.

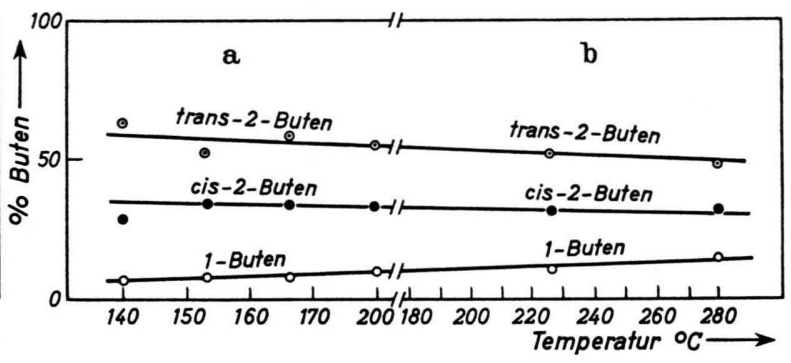

Abb. 4. Produktverteilung an $\mathrm{BeSO}_{4}$, a) für 2-Chlorbutan, b) für 1-Chlorbutan.

dar, die im wesentlichen alle auftretenden Typen der Produktverteilung umfassen. Generell nähert sich die Produktverteilung mit steigender Temperatur dem Gleichgewicht.

\section{2-Brombutan und 2-Chlorbutan}

Wie man sieht, ist im unteren Temperaturbereich bei einigen Katalysatoren, z. B. $\mathrm{Li}_{2}\left(\mathrm{SO}_{4}\right)$ (Abb. 1 ) und $\mathrm{BaSO}_{4}$ (Abb.2) das cis-Buten vor dem trans-2- 


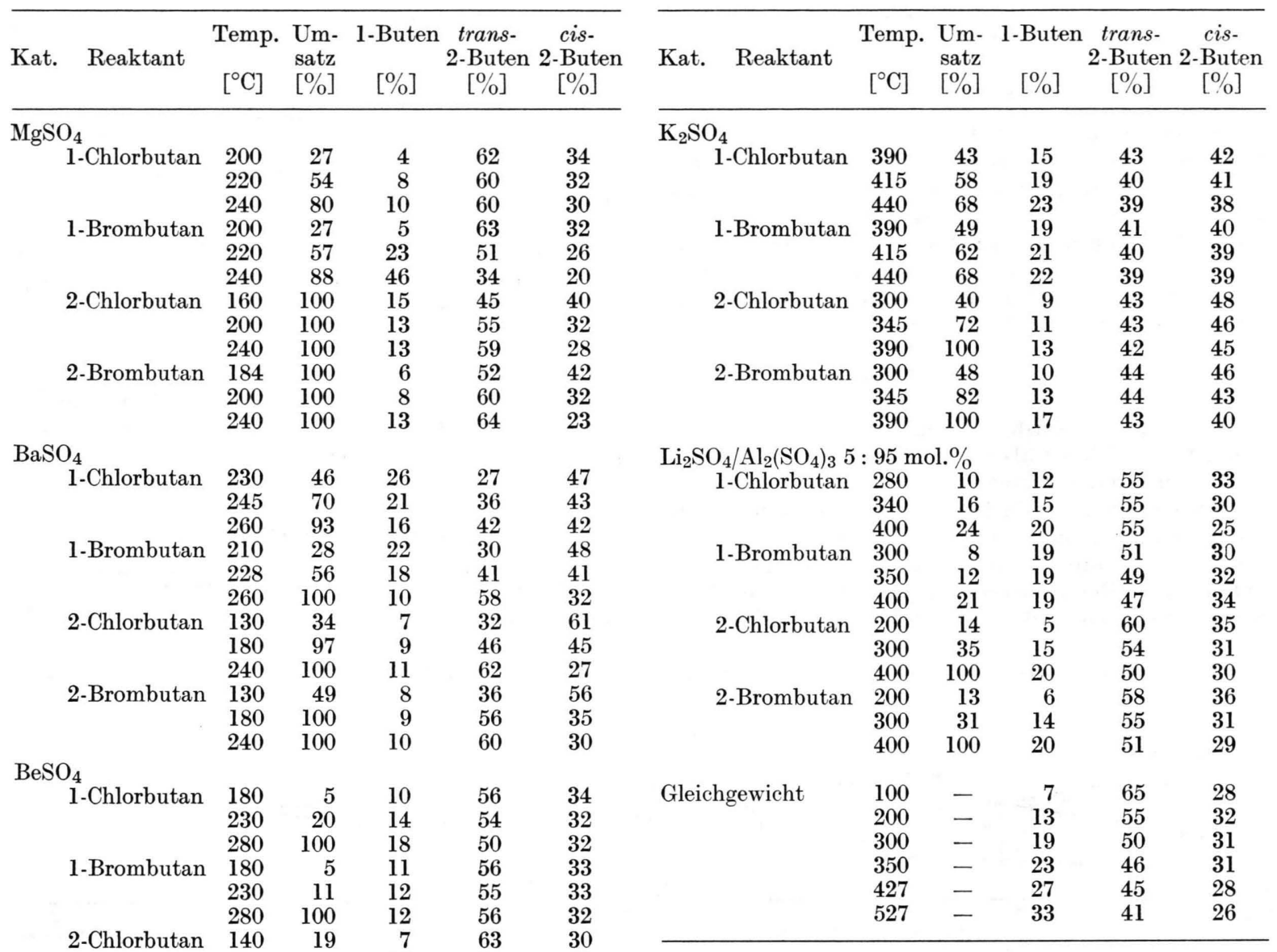

Tab. 1. Umsätze und Produktverteilungen bei der Eliminierung sowie Gleichgewichtswerte der drei linearen Butene.

* Der zu 100\% fehlende Rest, entspricht einem nicht identifizierten Produkt.

$\mathrm{Al}_{2}\left(\mathrm{SO}_{4}\right)_{3}$

$\begin{array}{lrrrrr}\text { 1-Chlorbutan* } & 240 & 17 & 7 & 60 & 33 \\ & 320 & 41 & 20 & 41 & 26 \\ \text { 1-Brombutan* } & 390 & 100 & 28 & 24 & 17 \\ & 330 & 8 & 18 & 58 & 34 \\ \text { 2-Chlorbutan } & 390 & 100 & 22 & 39 & 22 \\ & 200 & 42 & 13 & 56 & 12 \\ & 240 & 65 & 14 & 52 & 34 \\ \text { 2-Brombutan } & 270 & 100 & 16 & 50 & 34 \\ & 220 & 32 & 11 & 53 & 36 \\ & 250 & 95 & 15 & 51 & 34 \\ & 270 & 100 & 15 & 50 & 35\end{array}$

$\mathrm{Li}_{2} \mathrm{SO}_{4}$

$\begin{array}{lrrrrr}\text { 1-Chlorbutan } & 360 & 2 & 100 & - & - \\ & 390 & 10 & 100 & - & - \\ & 420 & - & 100 & - & - \\ \text { 1-Brombutan } & 320 & 2 & 100 & - & - \\ & 370 & 19 & 100 & - & - \\ & 390 & 28 & 88 & 12 & - \\ \text { 2-Chlorbutan } & 270 & 10 & 15 & 27 & 58 \\ & 330 & 39 & 19 & 32 & 49 \\ & 390 & 100 & 22 & 40 & 38 \\ \text { 2-Brombutan } & 230 & 45 & 13 & 29 & 58 \\ & 330 & 100 & 18 & 43 & 39 \\ & 390 & 100 & 20 & 52 & 28\end{array}$

Buten stark bevorzugt. Diese Produktverteilung entspricht nicht dem Gleichgewicht. Das bevorzugte Primärprodukt ist nicht das thermodynamisch stabilste (vgl. die Gleichgewichtsverteilungen, die in der Tabelle angegeben sind).

Höchstwahrscheinlich ist die hier gefundene Produktverteilung nicht genau die Primärprodukt-Verteilung. Was wir jedoch sagen können, das ist, daß die wirkliche Primärverteilung mindestens so weit, höchstwahrscheinlich aber weiter vom Gleichgewicht entfernt liegen muß als die hier angegebene Verteilung. Die Aktivierungsenergie für die Bildung von cis-2-Buten muß somit niedriger liegen als die für die beiden anderen Butene. Wir haben früher ${ }^{2}$ eine Erklärung dafür vorgeschlagen, die von den Stabili-

1 G. u. I. Martín, noch unveröffentlichte Messungen, Caracas 1966.

${ }^{2}$ H. Noller, W. Löw u. P. Andrév, Ber. Bunsengesellsch. physik. Chem. 68, 663 [1964]. 
tätsverhältnissen der Konformationen des intermediären Carbonium-Ions ausgeht. Als Alternative kann man jedoch auch an sterische Hinderungen am Katalysator denken, die bei der Bildung des cisOlefins weniger ins Gewicht fallen als bei der Bildung des trans-Olefins.

Vergleicht man nun das Ausmaß der cis-Bevorzugung bei den einzelnen Katalysatoren, so erhält man den Eindruck, daß diese zunimmt, wenn die Aktivität (s. Tabelle in der vorhergehenden Mitteilung) des Katalysators abnimmt. An den Katalysatoren mit zwei- und dreiwertigen Kationen wird eher trans-2-Buten bevorzugt (Abbn. 4 und 5), am wenigsten an $\mathrm{BaSO}_{4}$ (Abb. 2). $\mathrm{MgSO}_{4}$ bevorzugt je nach der Behandlung das cis- oder das trans-Olefin (vgl. Abb. 6 und Tabelle, s. weiter unten). Die Katalysatoren mit einwertigen Kationen, insbesondere $\mathrm{Li}_{2} \mathrm{SO}_{4}$, zeigen cis-Bevorzugung (Abb. 1).

Wie eine neue Untersuchung zeigt ${ }^{3}$, ist diese cisBevorzugung an $\mathrm{Li}_{2} \mathrm{CO}_{3}$ und $\mathrm{Li}_{3} \mathrm{PO}_{4}$ noch stärker ausgeprägt.

$\mathrm{Da}$ der aktivere Katalysator die geringere cisBevorzugung zeigt, hängt zu einem Teil wohl damit zusammen, daß am aktiveren Katalysator auch die sekundäre (als Folgereaktion ablaufende) Isomerisierung der Butene zum Gleichgewicht rascher verläuft. Ein wesentlicher Grund aber dürfte auch der sein, daß der aktivere Katalysator in der Regel die Aktivierungsenergien aller an ihm möglichen Reaktionen stärker herabsetzt als der weniger aktive. Etwas Ähnliches wird man für die Aktivierungsenergien aller zu einem Reaktionsakt gehörenden Teilschritte sagen dürfen. Der aktivere Katalysator hat sowohl für die Bildung des intermediären Carbonium-Ions die kleinere Aktivierungsenergie wie auch für die sich von diesem aus bildenden Butene. Da der Katalysator die Aktivierungsenergien, verglichen mit der Homogenreaktion oder auch mit einem wenig aktiven Katalysator, wohl nicht um den gleichen Absolutbetrag, sondern wahrscheinlich etwa im gleichen Verhältnis herabgesetzt, so vermindern sich am aktiven Katalysator die Differenzen aller Aktivierungsenergien. Dies hat zur Folge, daß der aktivere Katalysator meist auch der weniger selektive sein wird. Denn Selektivität bedeutet ja nichts anderes als einen großen Unterschied in den (freien) Ak-

3 A. Ismayel, Trabajo Especial de Grado, Caracas 1966.

4 H. Noller, H. Hantsche u. P. Andréu, J. Catalysis 4, 354 [1965]; S. Serain, Trabajo Especial de Grado, Caracas 1965. tivierungsenergien für die Bildung der einzelnen Butene.

Möglicherweise kommt noch etwas weiteres dazu. An zweiwertigen Kationen haben wir höchstwahrscheinlich einen Mechanismus mit stark ausgeprägtem El-Charakter (d. h. einen Reaktionsverlauf über ein intermediäres Carbonium-Ion). Ein experimenteller Hinweis darauf ist das Fehlen jeglicher Stereospezifizität an solchen Katalysatoren ${ }^{4}$. An Katalysatoren mit einwertigen Kationen dagegen nimmt der El-Charakter des Mechanismus ab zugunsten des E2-Charakters (Synchronmechanismus), und zwar um so mehr, je basischer das Anion ist ${ }^{5}$. Dies könte bedeuten, daß an $\mathrm{Li}_{2} \mathrm{SO}_{4}$ und $\mathrm{K}_{2} \mathrm{SO}_{4}$ (Abbn. 1 und 3) der E2-Charakter höher ist als an zweiwertigen Sulfaten. Wenn dies zutrifft, dann müßte die stärkere cis-Bevorzugung mit dem höheren E2Charakter des Mechanismus zu tun haben. Eine endgültige Klärung dieser Frage steht noch aus.

Ein interessanter Effekt zeigt sich an $\mathrm{MgSO}_{4}$. Behandelt man den Katalysator bei $160^{\circ} \mathrm{C}$ vor und untersucht dann, ohne abzukühlen, die Produktverteilung bei dieser Temperatur, so findet man eine cis-Bevorzugung. Bei Temperaturerhöhung geht diese zunehmend in eine trans-Bevorzugung über. Auch dann, wenn man nach der Vorbehandlung bei $160{ }^{\circ} \mathrm{C}$ abkühlt und anschließend bei aufsteigender Temperatur die Produktverteilung bestimmt, so findet man cis-Bevorzugung (Abb.6).

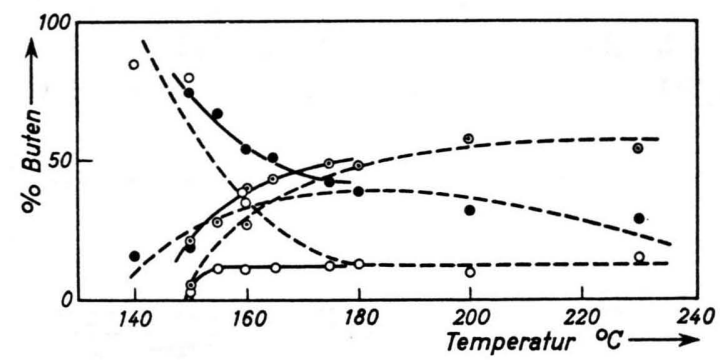

Abb. 6. Katalysator $\mathrm{MgSO}_{4}$. Vergleich von Eliminierung (durchgezogene Kurven) aus 2-Brombutan und Isomerisierung (gestrichelte Kurven) einer Mischung 1:1 von 1-Buten und HCl. $\bigcirc$ 1-Buten, $\odot$ trans-2-Buten, $\bullet$ cis-2-Buten.

Behandelt man dagegen den Katalysator bei $240{ }^{\circ} \mathrm{C}$ vor und bestimmt dann die Produktverteilung, ohne zuvor abzukühlen, so findet man bis herab zu $140{ }^{\circ} \mathrm{C}$ trans-Bevorzugung (diesem Ver-

5 O. Neufang, Dissertation, München 1967; J. Girón, noch unveröffentlichte Messungen, Caracas 1966. 
halten entsprechen die Angaben in der Tabelle). Wird bei dieser zuletzt geschilderten Verfahrensweise die Injektion bei $160^{\circ} \mathrm{C}$ mehrmals wiederholt, so geht die trans-Bevorzugung in eine cis-Bevorzugung über.

Worauf dieses Verhalten zurückzuführen ist, läßt sich auf Grund der vorliegenden Resultate kaum schon sagen. Möglicherweise hat es mit dem Wassergehalt des Katalysators zu tun. $\mathrm{MgSO}_{4}$ gibt bei etwa $160{ }^{\circ} \mathrm{C}$ sechs von seinen insgesamt sieben Molekeln Wasser $a^{6}{ }^{6}$, das letzte Molekül wird bei 178 bis $285{ }^{\circ} \mathrm{C}$ abgegeben. Der geschilderte Befund verdient wohl eine getrennte Untersuchung, da er wahrscheinlich wertvolle Aufschlüsse über die Selektivität liefern wird.

\section{1-Brombutan und 1-Chlorbutan}

Wie bei früheren Untersuchungen ${ }^{2,7}$ an anderen Katalysatoren, finden wir nun auch an Sulfaten, daß man, von den 1-Butanderivaten ausgehend, etwa die gleiche Produktverteilung erhält, wie von den 2-substituierten Butanen aus (Abb. 2 bis 5). Was diesen Befund betrifft, so brauchen wir unserer früheren Deutung nichts hinzuzufügen, daß das zunächst entstehende 1-Carbonium-Ion zum stabileren 2-Carbonium-Ion isomerisiert, das dann mit den aus den 2-substituierten Butanderivaten gebildeten identisch ist. Ein überraschender neuer Befund ist, daß $\mathrm{Li}_{2} \mathrm{SO}_{4}$ in einem gewissen Temperaturbereich streng selektiv ausschließlich 1-Buten entstehen läßt (Abb. 7). $\mathrm{Li}_{2} \mathrm{SO}_{4}$ ist der erste Katalysator (von den bisher

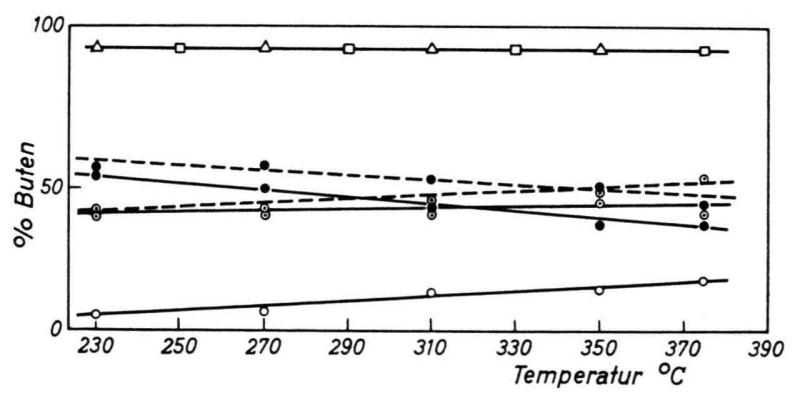

Abb. 7. Vergleich der Produktverteilung von Eliminierung und Isomerisierung an $\mathrm{Li}_{2} \mathrm{SO}_{4} . \triangle$ Eliminierung aus 1-Brombutan (es entsteht nur 1-Buten). $\square$ Isomerisierung von 1-Buten und $\mathrm{HCl} \mathrm{1:1} \mathrm{(im} \mathrm{ganzen} \mathrm{Bereich} \mathrm{keine} \mathrm{Isomerisie-}$ rung). Durchgezogene Linien Eliminierung aus 2-Brombutan, gestrichelte Linien Isomerisierung von cis-2-Buten und $\mathrm{HCl}$ 1:1. $\bigcirc$ 1-Buten, $\odot$ trans-Buten, $\bullet$ cis-2-Buten.

${ }^{6}$ C. Duval, Inorganic Thermogravimetric Analysis. Elsevier, Amsterdam 1963. untersuchten), der streng selektiv zu 1-Buten führt. Diese Eigenschaft läßt sich, wie wir kürzlich vorgeschlagen haben ${ }^{8}$, möglicherweise für die präparative Darstellung von 1-Buten ausnützen.

Inzwischen haben wir festgestellt, $\mathrm{da} \beta$ auch andere Lithiumsalze (z. B. $\mathrm{Li}_{2} \mathrm{CO}_{3}$ und $\mathrm{Li}_{3} \mathrm{PO}$ ) diese Eigenschaft haben, die offenbar zusammen mit einer ganz starken cis-Bevorzugung bei den 2-Derivaten des Butans auftritt ${ }^{3}$.

Ein stark ausgeprägter E2-Charakter des Mechanismus würde den Befund erklären. Wir kommen jedoch mit dieser Erklärung vorerst noch nicht recht durch. Denn dann müßten die Kaliumsalze diese Erscheinung noch stärker zeigen, oder zumindest ebenso. Denn an Kaliumsalzen sollte der E2-Charakter noch stärker sein, da doch die Wechselwirkung zwischen dem Kation des Katalysators und dem Halogen des Substrats kleiner ist als bei entsprechenden Lithiumsalzen. Kaliumsulfat aber zeigt weder die Bevorzugung von 1-Buten noch die von cis-2-Buten so stark wie Lithiumsalze (vgl. Tabelle und Abb. 3).

Andererseits aber ist die Stereospezifizität von Kaliumsalzen eher stärker als die von Lithiumsalzen mit dem gleichen Anion ${ }^{5}$. Nach der Stereospezifizität zu urteilen, haben somit die Kaliumsalze mehr E2Charakter, nach der Selektivität für 1- und cis-2Buten zu urteilen, haben die Lithiumsalze mehr E2Charakter. Offenbar ist hier noch ein weiterer Faktor im Spiel, den wir noch nicht kennen.

\section{Eliminierung und Isomerisierung}

Es ist nun interessant festzustellen, daß $\mathrm{Li}_{2} \mathrm{SO}_{4}$ auch nicht wie die anderen Katalysatoren 1-Buten zu isomerisieren vermag, wenn man es im Gemisch mit $\mathrm{HCl}$ über den Katalysator leitet. Wohl aber vermag $\mathrm{Li}_{2} \mathrm{SO}_{4}$ die Isomerisierung von cis- zu trans-2-Buten zuwegezubringen (Abb. 7).

Die Mehrzahl unserer Eliminierungs-Katalysatoren gibt auch die Isomerisierung der Butene, insbesondere im Beisein von $\mathrm{HCl}$. In den Abbn. 6 und 8 wird die Isomerisierung von 1-Buten mit der Eliminierung aus 2-Brombutan verglichen. An $\mathrm{MgSO}_{4}$ sind die Produktverteilungen bei hohen Temperaturen denen des Gleichgewichts ähnlich. Bei tiefen Temperaturen dagegen ist die Menge an 2-Buten bei einer Eliminierung größer als bei der Isomerisie-

7 H. Noller, P. Andrév, E. Schmitz, A. Zahlout u. R. BalLestros, Z. physik. Chem., Neue Folge 49, 299 [1966]. 


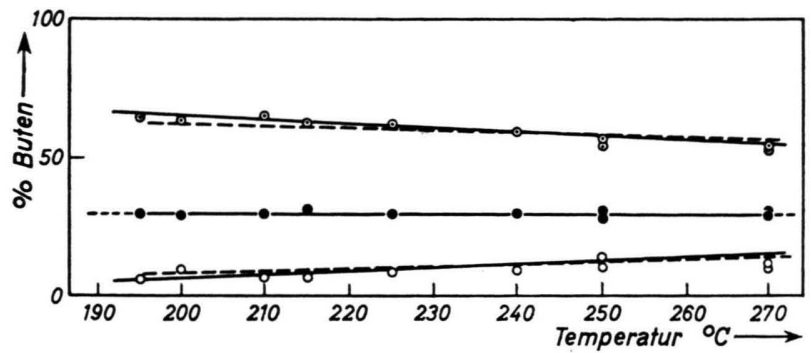

Abb. 8. Katalysator $\mathrm{Al}_{2}\left(\mathrm{SO}_{4}\right)_{3}$. Vergleich von Eliminierung (durchgezogene Kurven) aus 2-Brombutan und Isomerisierung (gestrichelte Linien) einer Mischung 1:1 von 1-Buten und HCl. $\bigcirc$ 1-buten, $\odot$ trans-2-Buten, $\bullet$ cis-2-Buten.

rung. Dies zeigt, daß die Bildung des 2-Butens bei der Eliminierung direkt erfolgt, auf keinen Fall über die intermediäre Bildung des 1-Butens. Wir haben zwar nie einen solchen Mechanismus vermutet, was aber kaum bedeuten kann, daß man diese Frage nicht zu prüfen hätte. Wenn man bedenkt, daß die Pyrolyse von 2-Chlorbutan höchstwahrscheinlich eine cis-Eliminierung ist - sie liefert 1-Buten als Hauptprodukt ${ }^{9}$ - so scheint ein solcher Reaktionsmechanismus nicht so abwegig. Die hier vorliegenden Resultate schließen ihn jedoch aus. Denn in diesem Fall müßte bei tieferen Temperaturen 1-Buten das Hauptprodukt sein, da die Gelegenheit zu der anschließenden Isomerisierung geringer ist, als wenn man 1-Buten und $\mathrm{HCl}$ selbst vorgibt. An $\mathrm{Al}_{2}\left(\mathrm{SO}_{4}\right)_{3}$ (Abb. 8) sind die beiden Produktverteilungen im gesamten Temperaturbereich einander gleich und sehr ähnlich dem Gleichgewicht. Dieser Fall erlaubt uns natürlich keinen ähnlichen Rückschluß auf den Mechanismus wie bei $\mathrm{MgSO}_{4}$.

Ein ähnlicher Vergleich für die Eliminierung aus 2-Brombutan und die Isomerisierung von cis-2-Buten ist in den Abbn. 7 und 9 für $\mathrm{Li}_{2} \mathrm{SO}_{4}$ bzw. $\mathrm{BaSO}_{4}$ als Katalysator dargestellt. In beiden Fällen ist die bei der Eliminierung erhaltene Olefinverteilung näher dem Gleichgewicht als die bei der Isomerisierung erhaltene. Dies läßt den Schluß zu, daß sich als Primärprodukt nicht ausschließlich cis-2-Buten bildet, das dann in Folgereaktion zu den anderen Olefinden isomerisiert, sondern daß die drei Olefine wenigstens zu einem Teil wirkliche Primärprodukte sind. Würde es sich um eine Isomerisierung in einer Folgereaktion handeln, so müßte die Mischung aus

8 H. Noller, M. Rosa-Brusin u. P. Andréu, Angew. Chem. 79, 187 [1967] ; Internat. Ed. 6, 170 [1967].

9 H. Heydtmann u. G. Rinck, Z. physik. Chem., Neue Folge 30, 25 [1961] ; 36, 75 [1963] ; A. Maccoll u. R. H. Stone, J. chem. Soc. [London] 1961, 2756.

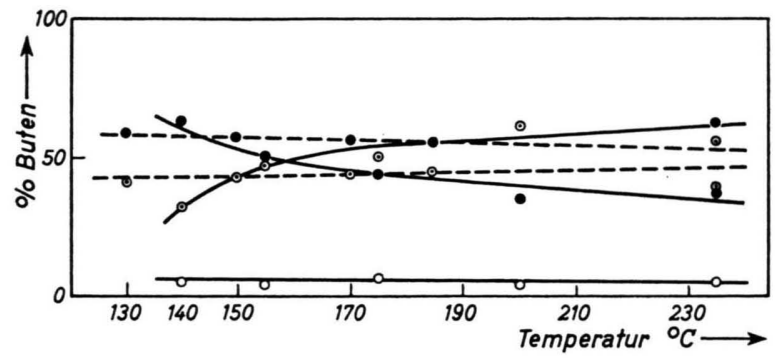

Abb. 9. Katalysator $\mathrm{BaSO}_{4}$. Vergleich von Eliminierung (durchgezogene Kurven) aus 2-Brombutan und Isomerisierung (gestrichelte Kurven) einer Mischung $1: 1$ von cis-2-Buten und HCl. $\bigcirc$ l-Buten, $\odot$ trans-2-Buten, $\bullet$ cis-2-Buten.

cis-2-Buten und $\mathrm{HCl}$ ein näher beim Gleichgewicht liegendes Olefingemisch geben, da sich ja bei der Eliminierung die zur Isomerisierung befähigten Reaktanten erst bilden müssen.

In Abb. 10 ist ein analoger Vergleich zwischen 1-Brombutan und der Mischung aus 1-Buten und $\mathrm{HCl}$ für $\mathrm{BaSO}_{4}$ als Katalysator dargestellt. Auch in diesem Fall tritt die Frage auf, ob sich zunächst 1-Buten bildet, das desorbiert und in einer neuen Adsorption zu 2-Buten isomerisiert oder ob sich

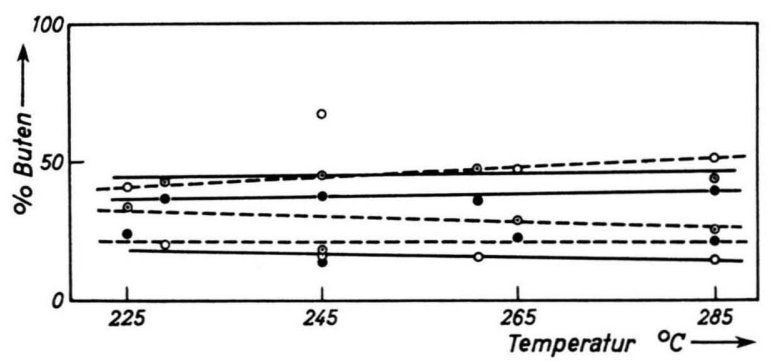

Abb. 10. Katalysator $\mathrm{BaSO}_{4}$. Vergleich der Produktverteilung von Eliminierung (durchgezogene Kurven) aus 1-Brombutan und Isomerisierung (gestrichelte Kurven) einer $\mathrm{Mi}$ schung $1: 1$ von 1 -Buten und HCl. $\bigcirc$ l-Buten, $\odot$ trans-2-Buten, $\bullet$ cis-2-Buten.

2-Buten gleich in dem zur ersten Reaktion führenden Adsorptionsakt bildet. Wie man sieht, bleibt die 2-Butenbildung bei der Isomerisierung der Mischung von 1-Buten und $\mathrm{HCl}$ weit hinter der der Eliminierung zurück, was nur dann verständlich ist, wenn sich 2-Buten nicht in einem Folgeschritt bildet, sondern als Primärprodukt, d.h. wenn die Bildung des zur Isomerisierung fähigen Carbonium-Ions und dessen anschließende Isomerisierung im gleichen Adsorptionsakt erfolgen.

Wir danken dem Consejo de Desarrollo Científico y Humanístico dafür, daß er die Besoldung eines der Autoren (P.A.) übernommen und außerdem diese Arbeit materiell unterstützt hat. 